IWSS

Information Systems

/ INS INformation Systems

NS Cumulative effective Hölder exponent based indicator for real time fetal heartbeat analysis during labour

Z.R. Struzik, W.J. van Wijngaarden

RePORt INS-R01 10 NOVember 30, 2001 
CWI is the National Research Institute for Mathematics and Computer Science. It is sponsored by the Netherlands Organization for Scientific Research (NWO).

$\mathrm{CWI}$ is a founding member of ERCIM, the European Research Consortium for Informatics and Mathematics.

CWI's research has a theme-oriented structure and is grouped into four clusters. Listed below are the names of the clusters and in parentheses their acronyms.

Probability, Networks and Algorithms (PNA)

Software Engineering (SEN)

Modelling, Analysis and Simulation (MAS)

Information Systems (INS)

Copyright @ 2001, Stichting Centrum voor Wiskunde en Informatica

P.O. Box 94079, 1090 GB Amsterdam (NL)

Kruislaan 413, 1098 SJ Amsterdam (NL)

Telephone +31205929333

Telefax +31 205924199 


\title{
Cumulative Effective Hölder Exponent Based Indicator for Real Time Fetal Heartbeat Analysis During Labour
}

\author{
Zbigniew R. Struzik \\ CWI \\ P.O. Box 94079, 1090 GB Amsterdam, The Netherlands \\ email: Zbigniew.Struzik@cwi.nl \\ Willem J. van Wijngaarden \\ Afdeling Verloskunde en Gynaecologie, H4 - 205 \\ Academisch Medisch Centrum \\ Postbus 22660, 1100 DD Amsterdam, The Netherlands \\ email: W.J.vanWijngaarden@amc.uva.nl
}

\begin{abstract}
We introduce a special purpose cumulative indicator, capturing in real time the cumulative deviation from the reference level of the exponent $h$ (local roughness, Hölder exponent) of the fetal heartbeat during labour. We verify that the indicator applied to the variability component of the heartbeat coincides with the fetal outcome as determined by blood samples. The variability component is obtained from running real time decomposition of fetal heartbeat into independent components using an adaptation of an oversampled Haar wavelet transform. The particular filters used and resolutions applied are motivated by obstetricial insight/practice. The methodology described has the potential for real time monitoring of the fetus during labour and for the prediction of the fetal outcome, allerting the attending staff in the case of (threatening) hypoxia.

2000 Mathematics Subject Classification: 28A80, 68T10, 68P10

1999 ACM Computing Classification System: H1, I5, Jm, J2, E2

Keywords and Phrases: fetal heartbeat, real time monitoring, multi-resolution, heartbeat variability, Hölder exponent

Note: a full colour version of this paper can be downloaded from www.cwi.nl/ ${ }^{\sim}$ zbyszek
\end{abstract}

\section{INTRODUCTION}

Methods of wavelet transform modulus maxima (WTMM) based multifractal analysis (MF) and detrended fluctuation analysis (DFA) have been demonstrated to be suitable for capturing scaling and correlation characteristics of the fluctuations in human heartbeat intervals $[1,2,3,4]$. These characteristics, obtained under a variety of conditions, have also been shown to reflect deviations in the heartbeat due to a variety of malfunctions $[5,6,4,7]$ and physiological behaviour $[8,9,10]$. Obviously, these results can be considered for clinical applications.

Unfortunately, such standard methods of statistical analysis of heartbeat signals are not directly applicable to the practical problem of evaluating (the characteristics of) the fetal heartbeat in real time during labour.

There are several reasons for this. One is that statistical techniques use long stretches of data to provide estimates of global measures (entities), like correlation exponents or multifractal spectra. A typical measurement requires over 30,000 samples $\left(2^{15}\right)$ to provide reliable estimates of scaling for the extraction of exponents and reliable transformation from scaling exponents to the domain of multifractal spectra. 
The typical heartrate of a fetus is about 130 beats per minute. The required time stretch for acquiring a sufficiently long data set $\left(2^{15}\right)$ is thus about 250 minutes. By this time, the baby is often already born. Decisions about an intervention (such as a Caesarean section) have to be taken on the basis of $10-60$ minutes long observations of the heartbeat. ${ }^{1}$

In addition to this ${ }^{2}$ correlation exponents and multifractal spectra derived from them are rather sensitive to spikes, missing data, noise bursts and boundary effects. ${ }^{3}$ Such erroneous data can cause a dramatic alteration of the results, which is particularly bad due to the lack of indication of where the errors come from - a problem inherent to global statistical techniques. Fetal heartbeats during labour are recorded in difficult circumstances and subject to frequent data fall out and a high level of noise. Missing data in fetal heartbeat records can amount to about $20-40 \%$. This does not include spikes and other noise.

Another serious problem for standard methods of analysis is the presence of the so-called decelerations in fetal heartbeat. These are sudden drops in the heartrate of the fetus, which can be caused by a number of events such as compression of the umbilical cord, increased intracranial pressure of the fetus during contractions or (temporary) hypoxia. The deficiency of oxygen caused by the contractions is compensated for by slowing down the heartrate (the so-called whale effect). Thus the presence of these $20-200$ beats long drops in the heartrate does not necessarily imply a severe condition of hypoxia. However, they severely distort any standard method of heartrate analysis - the amplitude of the decelerations can be about one degree of magnitude larger than that of the residual fluctuations of the heartbeat.

Lastly, but importantly, global statistical techniques are usually computationally expensive. However, the most unsuitable for real time applications is the lack of update mechanism on new samples. Taken as they are, such techniques would require recalculation of all the coefficients of the time series on each new sample - an unacceptable approach even in the days of cheap computational power.

Although these constraints practically disqualify standard methods from being used in medical practice, adaptations of standard approaches are possible. We have, therefore, considered the abovementioned problems and designed a methodology which is capable of providing the required characteristics of fetal heartbeat in real time.

The steps taken were thus the design of a running, incremental real time decomposition scheme [13], which is capable of separating meaningful components of the fetal heartbeat. The criterium for the decomposition used here is not arbitrary (or driven by properties of the decomposition) as in standard wavelet decomposition, but designed to capture the unique features of the fetal heartbeat. These features are meant to reflect the components of the heartbeat which are analysed by obstetricians in standard clinical practice. The effect of such a decomposition is that the high frequency variability component can be analysed separately from the deceleration component. In this way, the influence of decelerations on the spectrum of exponents can be minimized. Additionally, this way of decomposing the signal gives the possibility of providing the obstetrician with estimates reflecting traditionally observed entities.

Instead of the standard correlation analysis on long stretches of data, a simplified (effective) Hölder exponent estimate $h$ has been used [14]. It has been proven to provide useful roughness characteristics in the context of heartbeat or financial analysis $[10,15]$. In this work, we use it to analyse the local roughness of the variability component. This is a novel approach in obstetrics, since the standard way of estimating variability level refers to amplitude sensitive standard deviation rather than scale-free characteristics like the local roughness exponent.

By introducing a special cumulative indicator, we have been able to verify that the increase of the exponent $h$ (local roughness exponent) of the variability component of the fetal heartbeat relates to adverse blood gas values of the fetus (hypoxia condition). It has, therefore, a potential for monitoring

\footnotetext{
${ }^{1}$ It has to be noted that fetal heartbeat is the only indicator of the state of the fetus. Therefore the characteristics determined from it are crucial in taking the decision to carry out an operative delivery in case of suspected hypoxia.

${ }^{2}$ despite the fact that techniques like WTMM provide localised information,

${ }^{3}$ This can particularly affect higher moment calculations, resulting in serious errors in the MF spectrum. [11, 12]
} 
the fetus and for the prediction of the fetal outcome. This observation confirms the reported observations on adults, where the increase in the (global) correlation exponent corresponding to the loss of anti-correlation has been attributed to a number of malfunctions in the cardiac system. ${ }^{4}$

The contents of this paper are divided as follows. In section 2, we introduce a running time decomposition of the fetal heartrate (FHR). Most of the technology used here is self-contained, but the reader may want to check Ref. [13] for technical details of real time, Haar-type, wavelet decomposition. In section 3, the ideas of running multiresolution decomposition are used to separate meaningful components of the fetal heartbeat, as motivated by obstetricial insight/practice. In section 4, basic operators are introduced, which act on the decomposition coefficients. The operators reveal and enhance collective behaviour in the fluctuations of the coefficients. Finally, in section 5, a cumulative operator is defined, which is next applied to the effective Hölder exponent of the variability component of the fetal heartbeat. Section 6 closes the paper with conclusions and future plans.

\section{Running, Real time Decomposition Scheme}

In real time applications, fast, incremental decomposition methods are inevitable. The wavelet decomposition methods traditionally used for the multifractal analysis of signals [16,4] were developed for off-line analysis. They make use of the Gaussian kernel based Wavelet Transform Modulus Maxima (WTMM) decomposition in its multiscale (Continuous Wavelet Transform (CWT) - based) variant for maximum accuracy at the cost of extremely high redundancy and considerable computational expense.

In our approach, we use a limited, special purpose multiresolution decomposition of the signal with a simple block function as the smoothing kernel. ${ }^{5}$ Multiresolution approaches use a discrete ladder of resolution steps (usually dyadic) to represent the properties of the signal in possibly the least number of (orthogonal) coefficients [17, 18]. Often these ladder steps are chosen according to the orthogonality properties of the wavelet. The simplest, the Haar wavelet [19], requires a dyadic pyramid for complete and orthogonal decomposition of the input signal.

The space of the representation is partitioned by applying a smoothing kernel (block function in the case of the Haar wavelet) of a width, subsequently decreased by 2 (and the height renormalised to preserve L1 norm). The resulting multiresolution ladder contains approximations of the function obtained by smoothing the function (convolving with the smoothing kernel - the block function) in a stepwise increasing resolution. See figure 1 for an illustration of this principle.

The conversion to the multiresolution representation is now simple - the information content contained in one finer resolution step is subtracted from that contained in a less fine resolution level. In other words, the detail increase between each pair of subsequent resolution levels is what defines the multiresolution decomposition.

For our decomposition, we adopted a similar approach, but with several changes - firstly the number of approximation steps has been reduced to two. Secondly, the separation of both resolutions has been increased to one decade. Moreover, in the case of one of the resolution levels, we applied an adaptive filter which does not work at a fixed resolution. Its effective resolution is a result of the contents of the signal. The motivation for the particular choice of the primary approximation levels is the intention to separate the meaningful components of the fetal heartbeat according to the terminology traditionally used.

Another alteration to the dyadic decomposition scheme is the running approach, where the approx-

\footnotetext{
${ }^{4}$ It should be noted here that most malfunctions correspond with an insufficient supply of oxygen.

${ }^{5}$ One problem with the Gaussian is that it is defined on infinite support and therefore requires long sequences of coefficients to provide sufficient accuracy. Another problem is that in order to represent a relatively complex function like a Gaussian, one requires a large number of samples (at least five to ten for the Gaussian and more samples to represent its higher order derivatives). The variability features observed in heartbeat are of a resolution under 20 heartbeats. (This upper bound for the variability resolution comes from the presence of accelerations and decelerations). So while it is possible to perform single scale decomposition with a more complex function, it is not feasible to perform any realistic scaling analysis (a minimum of one decade scale span is required for this). Of course, a simple block function can be used down to the resolution of a single sample.
} 

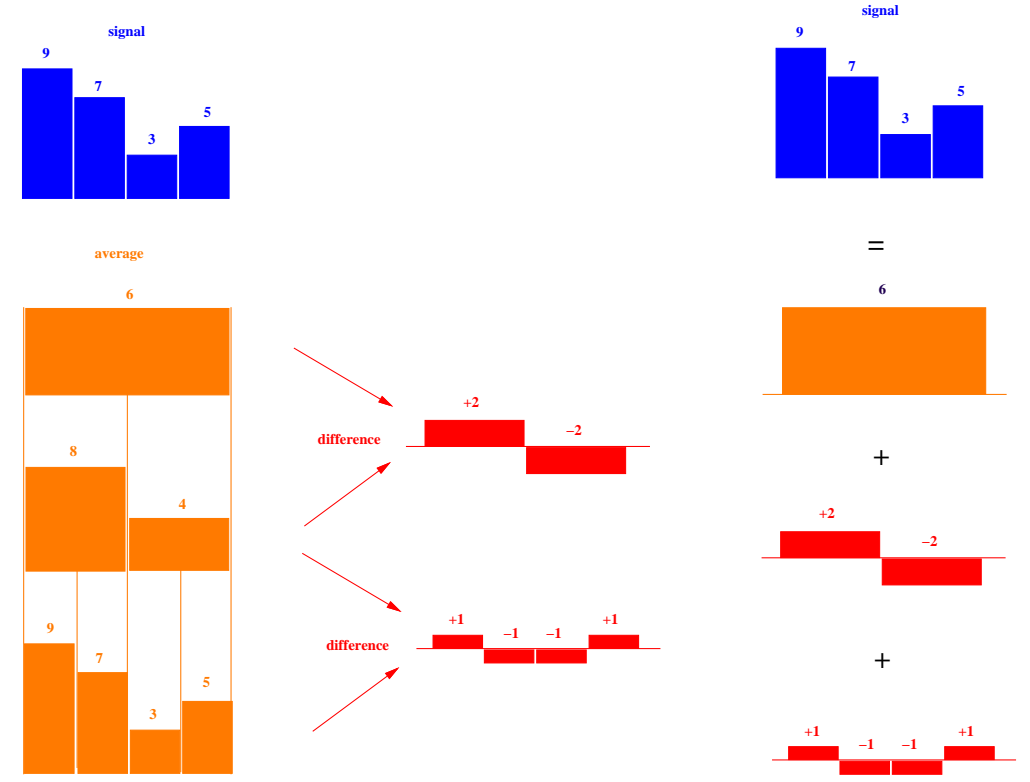

Figure 1: Decomposition of the example time series into Haar components. Right: reconstruction of the time series from the Haar components.

imation coefficients are obtained for each sample of the original signal. This, of course, resembles the continuous wavelet transform approach (we refer to it as an oversampled representation) and is simply obtained by convolving the filters with the input signal. Due to the fact that we use simple block smoothing filters, the operation is, in fact, a moving average of a certain block length. This scheme lends itself readily to incremental update on new samples, which in a more general setting is described in Ref. [13].

\section{Separating Meaningful Components of Fetal Heartbeat}

Fetal heartbeat is monitored during labour, as it is the only indicator of the well-being of the fetus. It is, therefore, used to alert the attending staff of possible hypoxia requiring direct intervention - a Caesarean section or $\mathrm{pH}$ and Base Excess estimation from a fetal scalp blood sample taken during labour. The heartbeat is usually analysed from the point of view of its three main characteristics:

- the level of the baseline - the low frequency outline of the heartbeat without spikes, high frequency variability and without decelerations and accelerations

- the presence and frequency of decelerations and accelerations

- the level of variability - the high frequency 'noise' of the heartbeat.

Using these characteristics, obstetricians can predict a good outcome very well. However, in cases of 'bad' fetal heartrate patterns, half the time the fetal outcome is good and an operative intervention may have been carried out unnecessarily. It turns out that we can conveniently approximate these characteristics in an appropriate decomposition.

As already mentioned in the previous section, we apply only two resolutions (scales) of approximation at the large separation of one decade (i.e. resolutions $a_{1}=200$ and $a_{2}=20$ ). The total decomposition plane is, therefore, partitioned into three sections, looking like the example in figure 2 .

The resolution $a_{1}=200$ beats is intended to approach the so-called baseline of the heartbeat - the low frequency 'backbone' of the fluctuations. This is where the adaptive filter proves necessary. The 

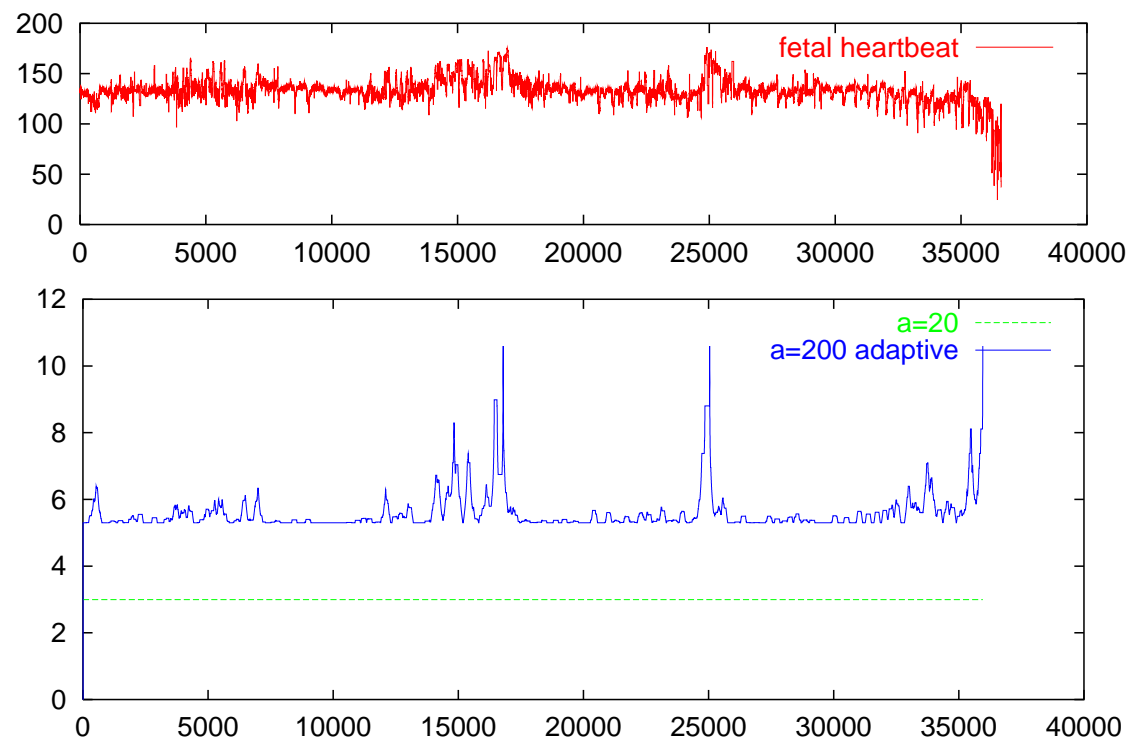

Figure 2: Original data above and the corresponding scales of decomposition below. Due to the adaptive nature of the smoothing filter, the exact appearance of the upper resolution level changes from time series to time series.

resolution of $a_{1}=200$ beats is suitable for approximating the baseline, but not in the presence of decelerations (or accelerations, sudden jumps of the heartbeat). The moving average filter performing approximation of the heartbeat at $a_{1}=200$ beats is thus equipped with a threshold mechanism which does not accept jumps or drops larger than one standard deviation from the (historic $N$ samples) mean value of the signal.

$$
\operatorname{MAA}_{\mathrm{a}_{1}}\left(f_{i}\right)=\frac{1}{a_{1}} \sum_{i=-a_{1} / 2}^{i=a_{1} / 2}\left(f_{i}\right) \delta(i)
$$

where

$$
\delta(i)= \begin{cases}1 & \text { for }\left(f_{i}-\operatorname{mean}\left(f_{i}, i=i-N, \ldots, i\right)\right)<\operatorname{stdev}\left(f_{i}, i=i-N, \ldots, i\right) \\ 0 & \text { otherwise }\end{cases}
$$

The second approximation level, centred at $a_{2}=20$ beats, uses a simple block smoothing function. It is intended to separate the highest frequencies, which we attribute to the so-called variability component.

$$
\mathrm{MA}_{\mathrm{a}_{2}}\left(f_{i}\right)=\frac{1}{a_{2}} \sum_{i=-a_{2} / 2}^{i=a_{2} / 2}\left(f_{i}\right)
$$

Just like in the standard wavelet decomposition, the multiresolution bands of decomposition are obtained from subtracting the multiresolution approximations at subsequent resolutions. As we have used only two levels of approximation, three bands of resolution are obtained: 

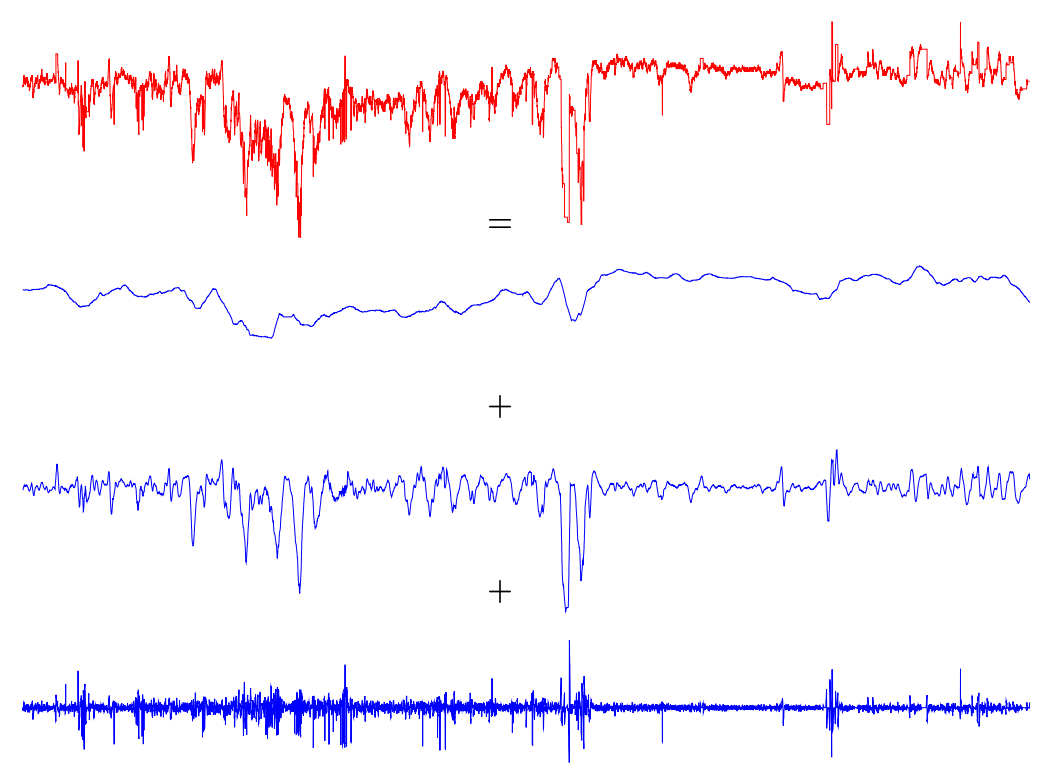

Figure 3: The decomposition of the fetal heartbeat time series. From top to bottom, the original time series, the low frequency baseline, the combined acceleration-deceleration line, the variability residue. All three components are orthogonal and can be summed up to give the original.

1. The first band of our decomposition is the entire low frequency component separated by the adaptive MA filter at the $a_{1}=200$ resolution level. It captures features of resolution less than $a_{1}=200$ and amplitude less than one standard deviation from the (historic) mean value of the signal. The filter used to define the baseline approximation level, Eqn. 3.1, is a low-pass filter, therefore the entire resolution band of low frequencies - the baseline, simply becomes:

$$
B_{i}=\operatorname{MAA}_{200}\left(f_{i}\right)
$$

2. The second band is obtained from subtracting the baseline approximation level from the variability approximation level. This contains the middle range of frequencies, capturing combined accelerations and decelerations.

$$
A D_{i}=\mathrm{MA}_{20}\left(f_{i}\right)-\mathrm{MAA}_{200}\left(f_{i}\right)
$$

3. Finally, the variability range of resolutions is obtained by subtracting the original time series from the approximation at the resolution level $a_{2}=20$. This variability is thus the signal, less baseline fluctuations, and less the decelerations and accelerations component.

$$
V_{i}=f_{i}-\mathrm{MA}_{20}\left(f_{i}\right)
$$

The result of applying such a procedure is a complete orthogonal decomposition into three components of the time series: the baseline, the combined line of accelerations and decelerations, plus the residual variability component. Of course, the original time series can be restored by simply summing up all three components, see figure 3 . 


\section{Constructing Meaningful Real time Indicators}

The components of the fetal heartbeat thus obtained carry information, which is (visually) analysed by the obstetrician. In order to mimic a visual evaluation of the trends and collective behaviour of the fluctuations and features in the decomposition, we apply running $M A_{1000}$ filters.

The local average effective acceleration/deceleration obtained in this way, see figure 4, indicates the effective level of deceleration which can be monitored and evaluated on-line. We will, however, not further pursue here the discussion of the relevance of this characteristic, leaving it to a separate communication. Rather, we intend to focus exclusively on the diagnostic capabilities of variability, or rather its roughness exponent.
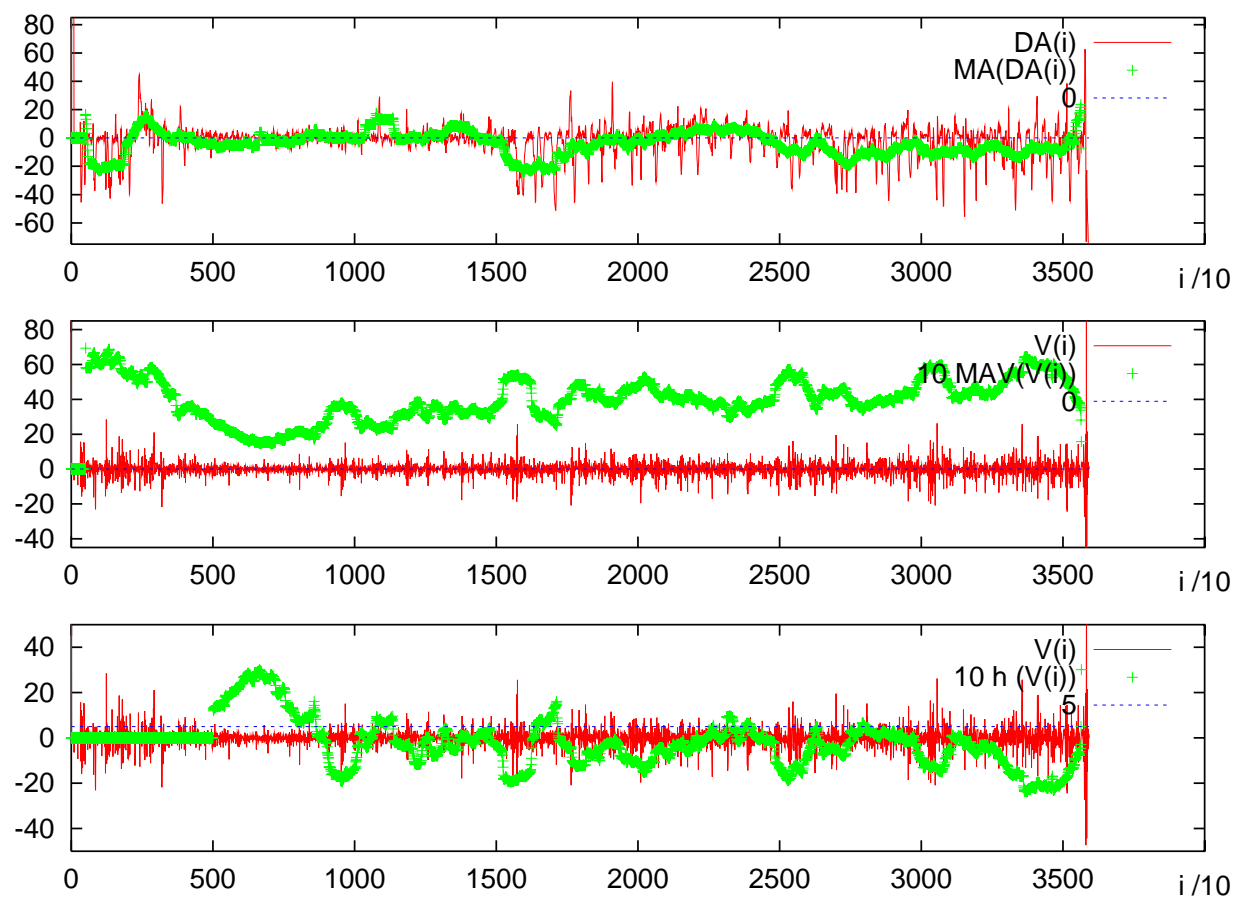

Figure 4: Real time indicators, from top to bottom: local average dec-/acceleration $M A_{1000}\left(D A_{i}\right)$, local average variability $M A V_{1000}\left(V_{i}\right)$, local 'Hölder' exponent $h_{e f f}\left(V_{i}\right)$. Both the $M A V_{1000}\left(V_{i}\right)$ and $h_{e f f}\left(V_{i}\right)$ have been rescaled by a factor 10 .

One could be tempted to apply a similar moving average filter to the variability component. Of course, this time it would have to be applied to the square amplitude, as the variability is usually perceived as the (mean) amplitude of high frequency deviations:

$$
\operatorname{MAV}_{\mathrm{a}}\left(V_{i}\right)=\sqrt{\frac{1}{a} \sum_{i=-a / 2}^{i=a / 2} V_{i}^{2}} .
$$

Such a fixed resolution indicator (for example at $a=1000$ ) would perhaps be considered an adequate measure of variability. However, it is known that our visual system picks scale-free characteristics when evaluating measures of roughness or variability. Therefore, even from the point of view of a visual evaluation, a fixed scale measure is not necessarily the best indicator.

It has been demonstrated [1-10] that scale-free measures of variability may be more suitable for 
diagnostic purposes. ${ }^{6}$ There are a number of methods providing scale-free characteristics, most of them are, however, unsuitable for real time local analysis of non-stationary time series. Therefore, for this study we use an adaptation of the effective Hölder exponent measure of local roughness [14, 11]. The original concept has already been used in the study of adult heartbeat, using the WTMM representation [10].

The local effective roughness of the variability component $V$ is defined as the logarithmic increase of the (standard deviation) of variability across scales/resolutions:

$$
h_{e f f}\left(V_{i}\right)=\frac{\log \left(\operatorname{MAV}_{\mathrm{a}_{1}}\left(V_{i}\right)\right)-\log \left(\operatorname{MAV}_{\mathrm{a}_{\mathrm{h}}}\left(V_{i}\right)\right)}{\log \left(a_{l}\right)-\log \left(a_{h}\right)}
$$

Two relatively rough resolutions $\left(a_{h}=1000\right.$ and $\left.a_{l}=10000\right)$ have been used for the evaluation of the local $h^{7}$ Also, we have used a simplification of the local effective exponent concept from Ref. $[14,11]$, in that a simple block function (moving average filter) is used instead of the wavelet. Using the moving average filters makes possible incorporation of the local $h$ evaluation in the real time incremental decomposition framework, as described above. Of course the local $h$ analysis with smoothing block kernels is only possible due to the fact that the variability component $V$ (as defined in Eqn. 3.5) is effectively free of any trends.

\section{Cumulative Hölder Exponent Based Real time Indicator}

There is no reason why the local Hölder exponent of the variability $h\left(V_{i}\right)$ should be stationary. It reflects dynamic changes in the condition of the fetus and the degree of stress to which it is subjected. Despite the fact that stress has a rapid effect on the heartbeat, the effects on the state of the fetus are not always immediate. This is why short dynamic changes in heartbeat characteristics may not be relevant and not representative of the state of the fetus.

Rather than using a long observation window, which would capture trend behaviour, we use a cumulative indicator, which still has the resolution of the mean local $h$ used in the previous section. The cumulative $h$ is defined from the beginning of the observation and with respect to some normal reference level $h_{\text {ref }}$ :

$$
h_{\text {cum }}\left(V_{i}\right)=-\sum_{l=1}^{i}\left(h_{e f f}\left(V_{l}\right)-h_{r e f}\right) .
$$

The minus sign is introduced to give the $h_{\text {cum }}$ indicator increasing direction when the level of local correlations is lower than $h_{\text {ref }}$. This corresponds with a healthy condition. The case of higher correlations is associated with problems and, therefore, the accumulation of a positive difference $\left(h_{e f f}\left(V_{l}\right)-h_{r e f}\right)$ will lead to decreasing cumulative $h$.

We have tested several examples of fetal heartbeats and found a good correlation with the fetal outcome, as determined by the blood tests. In figure 5, we plot six cases where three represent good outcomes and three bad outcomes. The cumulative indicator steadily increasing or remaining within some margin of fluctuations indicates no problems and a good prediction. When the indicator plunges down, it calls for intervention. This can, of course, happen at any moment during labour. The nature of this process is dramatically non-stationary, and a period of positive evaluation can be interrupted at any stage (for example by the occlusion of the umbilical cord due to movement). One of our examples (figure 5 lower right), shows the cumulative indicator plunging after a prolonged homeostasis.

For the sake of verification of the predictive capability of $h_{\text {cum }}$, let us compare it with similar cumulative measures, which one can define over $V_{i}$ and $A D_{i}$. The cumulative measure over the acceleration/deceleration line $A D_{i}$ is taken directly as:

\footnotetext{
${ }^{6}$ Of course, this does not exclude using amplitude based measures of variability in parallel.

${ }^{7}$ Note that the actual (temporal) resolution of the local $h$ so obtained is defined by the higher (finer) resolution level - the coarse reference resolution can be accumulated from past historic samples.
} 

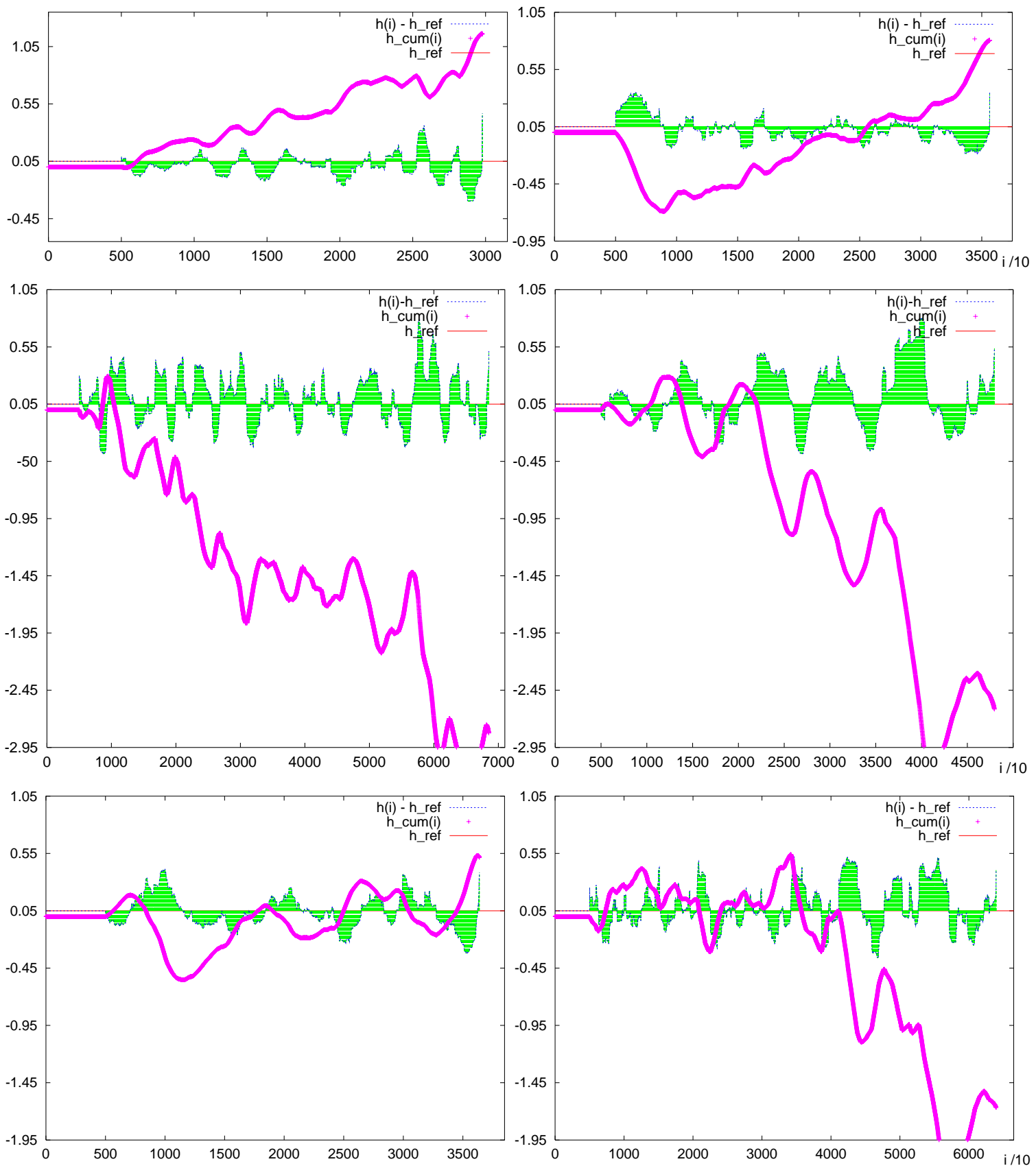

Figure 5: Six different time series analysed using the cumulative Hölder exponent based real time indicator. The time series correspond with three good outcomes (healthy) and three bad outcomes (hypoxia). The good outcomes can be identified by the indicator oscillating near zero or steadily increasing. In the case of hypoxia, the indicator plunges (down towards negative values). Problems can occur at any moment during labour, even after a stable condition, as is visible in the last of the six plots (lowest right). The first part of the plot is flat since this period is required for initialisation - acquiring a reference for the upper value for the Hölder exponent evaluation. Both the cumulative Hölder exponent $h_{\text {cum }}\left(V_{i}\right)$ (red line) and the deviation of the Hölder from the reference value $h_{\text {ref }}=0.05$ (blue filled curve) are plotted. The $h_{\text {cum }}\left(V_{i}\right)$ has been rescaled by a factor 0.01 . 


$$
\operatorname{cum}\left(A D_{i}\right)=-\sum_{l=1}^{i} A D_{l} .
$$

There is no need to introduce a threshold value in this measure, as $A D$ is defined with respect to ' 0 ' value as the reference - the boundary between the accelerations and decelerations.

The cumulative measure over variability $\operatorname{cum} V_{i}$, can be calculated without a threshold value, i.e. $V_{t h d}=0$, providing a measure of total variance accumulated during the process. A threshold value for variance can be introduced, although it is uncertain whether such an amplitude dependent reference value would be useful in practice.

$$
\operatorname{cum}\left(V_{i}\right)=-\sum_{l=1}^{i}\left(V_{l}-V_{t h d}\right)
$$

In order to analyse the temporal development of various indicators, we have plotted all three indicators for six different heartbeat time series in figure 6 . The indicators are plotted using a thick line in various colours. The colours used are: pink for cumulative thresholded variance $\operatorname{cum}\left(V_{i}\right)$, blue for cumulative collective acceleration/deceleration $\operatorname{cum}\left(A D_{i}\right)$ and green for cumulative thresholded Hölder exponent $h_{\text {cum }}\left(V_{i}\right)$. The threshold value $h_{\text {ref }}=0.05$, as before. A finer resolution range is used, namely $300-3000$ beats. The threshold value for variance has been set to $V_{t h d}=2.5$.

All these time series had been suspected of serious problems and all had blood samples taken. We have inluded plots of the actual time series for the reader to appreciate the immense diversity of features in these time series. The outcomes are described in a variety of ways. We have included an 'a posteriori' classification, given by an obstetrician, of the heartbeat records as 'ab-/normal'.

Also the Apgar scores at one AP1, five AP5 and ten AP10 minutes are given, indicating the condition of the child delivered. AP=9-10 means 'good', lower means 'bad'. Fetal blood samples base excess results are given as 'BE'. The less negative, the better. Less than -5 is rather bad. The same holds for blood samples taken after delivery - arterial denoted with artBE and ventricular venBE.

The diversity in the behaviour of the indicator is evident. On several occasions, the behaviour of the thresholded variance based indicator is very close to that of the roughness/Hölder exponent indicator. Still there are instances when the two diverge. The deceleration indicator differs most from both the variance and the Hölder exponent indicator.

There is no possibility of obtaining complete insight into the physiological condition of the fetus with one indicator, nevertheless we consider the results obtained with the Hölder exponent based indicator rather encouraging.

\section{Concluding Remarks}

Fetal heartbeat during labour is a highly non-stationary process, which needs to be monitored in real time.

We have presented a methodology of decomposition of fetal heartbeat into meaningful components suitable for real time monitoring of the fetus. We have also introduced a real time cumulative indicator, based on the effective Hölder exponent of the variability component of fetal heartbeat. The indicator has been demonstrated to provide insight into the highly non-stationary nature of the variability properites of the fetal heartbeat. It has also shown correlation with the blood samples, motivating its use as a monitoring and a predictive tool.

The exact parameters of the decomposition are subject to tuning. Also, other local measures of roughness of the variability component may provide better and more detailed insight. Combining the observations derived from (all) the components of the heartbeat (and possibly external knowledge) into a predictive tool may result in a substantially better predictive power. 

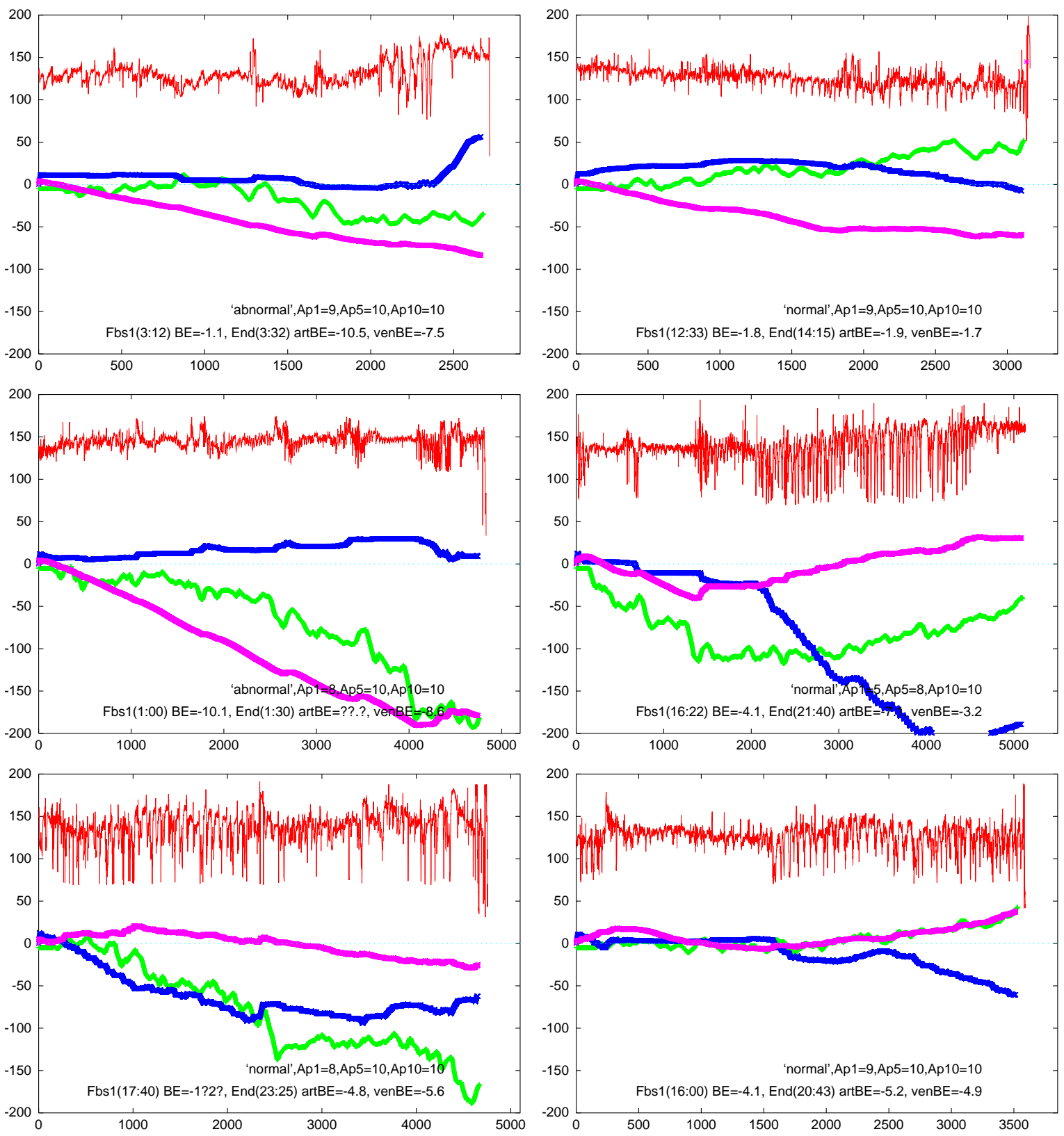

Figure 6: Six different time series analysed using three cumulative indicators with increased resolution of the $h$ exponent evaluation. The scale range taken is $300-3000$ beats. Colors used are: pink for cumulative thresholded variance $\operatorname{cum}\left(V_{i}\right)$, blue for cumulative collective acceleration/deceleration $\operatorname{cum}\left(A D_{i}\right)$ and green for cumulative thresholded Hölder exponent $h_{c u m}\left(V_{i}\right)$. In each figure, an 'a posteriori' doctor's classification of the heartbeat record is given as 'ab-/normal'. Also the Apgar scores at one AP1, five AP5 and ten AP10 minutes are given, indicating the condition of the child delivered. AP=9-10 means 'good', lower means 'bad'. Fetal blood samples base excess results are given as 'BE'. The less negative, the better. Less than -5 is rather bad. The same holds for blood samples taken after delivery - arterial denoted with artBE and ventricular venBE. 


\section{References}

1. M. Kobayashi, T. Musha, 1/f Fluctuation of Heartbeat Period, IEEE Trans Biomed. Eng., 29, 456-457 (1981).

2. C.-K. Peng, J. Mietus, J.M. Hausdorff, S. Havlin, H.E. Stanley and A.L. Goldberger Long-Range Anticorrelations and Non-Gaussian Bahavior of the Heartbeat Phys. Rev. Lett., 70, 1343-1346 (1993).

3. J. B. Bassingthwaighte, L. S. Liebovitch and B. J. West. Fractal Physiology, (Oxford University Press, 1994).

4. P.Ch. Ivanov, M.G. Rosenblum, L.A. Nunes Amaral, Z.R. Struzik, S. Havlin, A.L. Goldberger and H.E. Stanley, Multifractality in Human Heartbeat Dynamics, Nature 399, pp. 461-465, (1999).

5. R.G. Turccot, M.C. Teich, Fractal Character of the Electrocardiogram: Distinguishing Heartfailure and Normal Patients, Ann. Biomed. Eng. 24, 269-293 (1996).

6. M. Meyer, Scaling Properties of Heartbeat Interval Fluctuations in Health and Disease, in Fractals and Beyond, Ed. M.M. Novak, (World Scientific, 1998), pp. 33-42.

7. P.Ch. Ivanov, L.A. Nunes Amaral, A.L. Goldberger, S. Havlin, M.G. Rosenblum, H.E. Stanley and Z.R. Struzik, From 1/f Noise to Multifractal Cascades in Heartbeat Dynamics, Chaos 11 Special Issue on Unsolved Problems of Noise, Ed. Derek Abbott, to appear, (2001).

8. P. Ch. Ivanov, A. Bunde, L. A. N. Amaral, J. Fritsch-Yelle, R. M. Baevsky, S. Havlin, H. E. Stanley, and A. L. Goldberger, Sleep-wake Differences in Scaling Behavior of the Human Heartbeat: Analysis of Terrestrial and Long-term Space Flight Data, Europhys. Lett. 48, 594-600 (1999).

9. A. Bunde, S. Havlin, J.W. Kantelhardt, T. Penzel, J.-H. Peter and K. Voigt, Correlated and Uncorrelated Regions in Heart-rate Fluctuations During Sleep, Phys. Rev. Lett. 85, 3736 (2000).

10. Z. R. Struzik. Revealing Local Variablity Properties of Human Heartbeat Intervals with the Local Effective Hoelder Exponent. Fractals 9, No 1, (2001).

11. Z.R. Struzik, Determining Local Singularity Strengths and their Spectra with the Wavelet Transform, Fractals 8, No 2, (2000).

12. Z. R. Struzik, A. P. J. M. Siebes. Outlier Detection and Localisation with Wavelet Based Multifractal Formalism. Technical Report INS-R0008, CWI, Amsterdam, The Netherlands, (2000). Available from www.cwi.nl/ ${ }^{\sim}$ byszek 
13. Z. R. Struzik, Oversampling the Haar Wavelet Transform, CWI report INS-R0102, (2001). Available from www.cwi.nl/ ${ }^{\sim}$ zbyszek

14. Z. R. Struzik, Local Effective Hölder Exponent Estimation on the Wavelet Transform Maxima Tree, in Fractals: Theory and Applications in Engineering, Eds: M. Dekking, J. Lévy Véhel, E. Lutton, C. Tricot, Springer Verlag, pp. 93-112, (1999).

15. Z. R. Struzik, Wavelet Methods in (Financial) Time-series Processing, Physica A, 296 (1-2), 307-319, (2001).

16. A. Arneodo, E. Bacry and J.F. Muzy, The Thermodynamics of Fractals Revisited with Wavelets, Physica A, 213, 232-275, (1995).

J.F. Muzy, E. Bacry and A. Arneodo, The Multifractal Formalism Revisited with Wavelets, International Journal of Bifurcation and Chaos 4, pp. 245-302, (1994).

17. S. Mallat, A Theory for Multiresolution Signal Decomposition: The Wavelet Representation, IEEE Trans. PAMI, 11, pp. 674-693, (1989).

18. I. Daubechies, Ten Lectures on Wavelets. S.I.A.M., (1992).

19. A. Haar, Zur Theorie der orthogonalen Funktionensysteme, Math. Ann. 69, 331-71. (1910). 